\title{
134340 Pluto: nine years of CCD observations ${ }^{\star} \star \star$
}

\author{
C. H. Veiga
}

\author{
Observatório Nacional, Rua General José Cristino 77, Bairro Imperial de São Cristóvão Cep:20921-400 Rio de Janeiro, Brazil \\ Coordenação de Astronomia e Astrofísica \\ e-mail: cave@on.br
}

Received 24 March 2008 / Accepted 15 April 2008

\section{ABSTRACT}

\begin{abstract}
Aims. The purpose of this article is to present the new accurate astrometric CCD positions of Pluto, now designated as the minor planet 134340 Pluto and member of the Trans-Neptunian population. These positions were obtained from 1108 frames taken during 49 nights. The observations were distributed in 17 continuous missions between the years of 1995 and 2004.

Methods. The USNO-A2.0 star catalog, locally corrected by UCAC2 catalog, was used for the astrometric calibration. All positions were compared with those calculated by the PLU017 ephemeris.

Results. In this work precise positions of 134340 Pluto are presented. The residues, observed minus calculated positions, have mean and standard deviation smaller than $0{ }^{\prime} 05$, in the right ascension and declination coordinates' directions.
\end{abstract}

Key words. minor planets, asteroids - celestial mechanics - ephemerides - Kuiper Belt - astrometry

\section{Introduction}

Twelve years ago a systematic program of astrometric observations of the Jovian planets was started at the Laboratório Nacional de Astrofísica (LNA/MCT), Brazil (Veiga et al. 1995). The observations presented in this paper were made during 49 nights, within the period of 1995 to 2004, and 1108 CCD frames were obtained.

The results published here refer to the first 9 years of observation (no observations were made in the year 2000), when Pluto was still classified as a planet. Nowadays it is known as one of the three dwarf planet in the Solar System (International Astronomical Union - IAU resolution on September 13, 2006) (Gingerich 2006). This work was motivated by the recent discovery of two small moons of 134340 Pluto, Nix and Hydra, discovered May 15, 2005 by the Hubble Space Telescope (Weaver et al. 2006; Stern et al. 2006, 2007). The publication of new positions of 134340 Pluto can contribute to improved accuracy of the ephemeris and to a better understanding of the origin and formation of this quadruple system. On the other hand, these positions can also help the New Horizons spacecraft mission, which will arrive in 2015 at 134340 Pluto. The distribution of these CCD observations is shown in Fig. 1.

The next sections of this work describe the observations, the measurement method, and the data reduction procedures. The whole set of measured positions of 134340 Pluto are compared to the calculated positions using the JPL Development planetary Ephemeris-PLU017 (ICRF/J2000.0). Finally the result and conclusion are presented.

* Based on observations made at the Laboratório Nacional de Astrofísica/MCT-Itajubá-Brazil.

$\star \star$ Full Table 3 is only available in electronic form at the CDS via anonymous ftp to cdsarc.u-strasbg.fr (130.79.128.5) or via http://cdsweb.u-strasbg.fr/cgi-bin/qcat?J/A+A/486/613

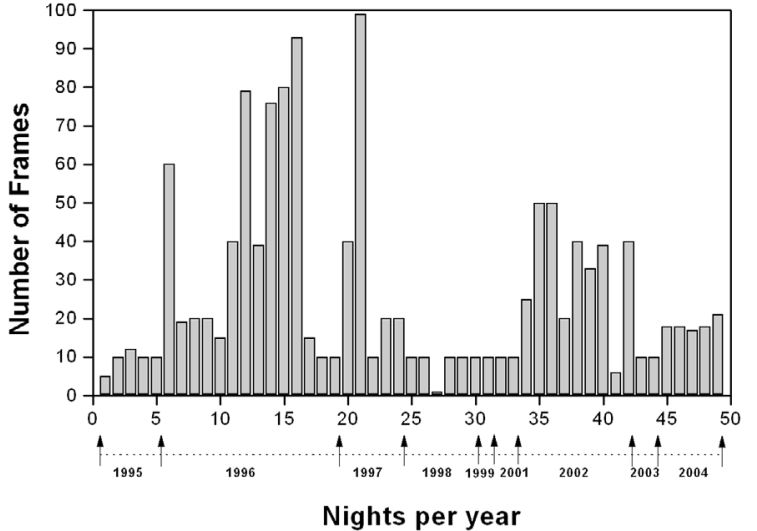

Fig. 1. The distribution of the observations with respect to time. Each bar corresponds to an observational night.

\section{Observations, measures, and astrometric calibration}

The 1108 observations were made at the $1.60 \mathrm{~m}$ Perkin-Elmer and $0.60 \mathrm{~m}$ Boller \& Chivens telescopes of the Laboratório Nacional de Astrofísica (LNA/MCT) Itajubá-Brazil. The local geographical coordinates for the telescopes are: Longitude $=$ $3^{\mathrm{h}} 02^{\mathrm{m}} 19^{\mathrm{s}} \mathrm{w}$, Latitude $=22^{\circ} 32^{\prime} 04^{\prime \prime} \mathrm{s}$ and Altitude $=1872 \mathrm{~m}$ (IAU reference number 874). The optical projects for both telescopes use the Ritchey-Chrétien system. The main parameters of two telescopes are given in Table 1.

Three CCD detectors were used to obtain the 1108 images of 134340 Pluto during the missions. In Table 2 the general characteristics of these devices are given. All CCDs are backilluminated types. No filters and also no adaptive optics system were used in the observations in order to correct the turbulence effect from Earth's atmosphere. The exposure time varied from 
Table 1. The parameters of two telescopes used in the observations of 134340 Pluto between 1995 and 2004.

\begin{tabular}{lll}
\hline \hline Primary mirror diameter & \multicolumn{1}{c|}{$\mathbf{1 . 6 0} \mathbf{~ m}$} & $\mathbf{0 . 6 0} \mathbf{~ m}$ \\
Scale in focal plane & $13^{\prime \prime} / \mathrm{mm}$ & $32^{\prime \prime} / \mathrm{mm}$ \\
Focal length & $15,6 \mathrm{~m}$ & $8,2 \mathrm{~m}$ \\
Cassegrain focal ratio & $\mathrm{f} / 10$ & $\mathrm{f} / 13,5$ \\
\hline
\end{tabular}

Table 2. The technical characteristics of the CCDs.

\begin{tabular}{ccc}
\hline \hline CCD description & Image size & Pixel size \\
\hline EEV CCD02-06-1-206 & $385 \times 578$ pixels & $22 \times 22 \mu \mathrm{m}$ \\
EEV CCD-05-20-0-202 & $770 \times 1152$ pixels & $22,5 \times 22,5 \mu \mathrm{m}$ \\
SITe SI003AB & $1024 \times 1024$ pixels & $24 \times 24 \mu \mathrm{m}$ \\
\hline
\end{tabular}

15 to $60 \mathrm{~s}$ depending on the following factors: the utilized detector sensibility, magnitudes of the star field, and mainly the meteorological conditions. The sky quality (seeing) varied between $0 . ' 7$ and 1.'2 for all missions.

To find the center of 134340 Pluto and star field images on the CCD, each position was determined by a two-dimensional Gaussian fitting a small circular area around the images. The non-personal measuring process was automated using an algorithm developed for Fortran compiler and the data plotting library Dislin by Veiga in 2007. This algorithm is based on the adjustment of a point-spread function, and a second-degree polynomial is also adjusted to remove a possible background contaminated by the light from the sky. Several observations were accomplished during nights of full Moon.

The binary system 134340 Pluto-Charon satellite is very close to each other. In the mean opposition, the maximum distance between them is less than 1.'0 and the differential visual magnitude is less than 2. In spite of their closeness, the relative measurement of the images' center can be corrected. The knowledge of the accurate relative orbit of Charon simplifies the correction. Then, this correction, described in Reinsch et al. (1994), Null et al. (1993), was used. The errors on the centering procedure were 0. '02 for 134340 Pluto and star images.

The classical process of astrometric calibration was used to determine the observed coordinates of the 134340 Pluto in the sky. In this process, the stars' USNO-A2.0 catalog (Monet et al. 1998) was used as a reference system. The UCAC2 cata$\log$ (Zacharias et al. 2004), which has shown astrometric precision better, was used to correct the local systematic errors of the USNO-A2.0 catalog.

The same stars in the UCAC2 catalog and USNO-A2.0 catalog were identified, in a field of 2 by 2 degrees. A least-square procedure was used with those positions based on a Householder transformation (Lawson \& Hanson 1974) to fit the transformation parameters. A second-degree polynomial was used to determine the USNO-A2.0 catalog star corrected coordinates. The characteristic standard deviation of the residuals for the stars from all the stellar fields was at $0{ }^{\prime} 11$.

Finally, using the star positions in the field of each CCD frame observation (measured and from the corrected catalog), a new least-square procedure was used to determine the parameters of the scale and orientation. The first-degree polynomial (six-constant model) was then used to determine the equatorial coordinates of 134340 Pluto.

In the list, available only in electronic form at the CDS, the topocentric observed positions of 134340 Pluto are referred to a mean equator and equinox J2000 system. The mean instant of observation is given in Julian Date (UTC) and right
Table 3. Sample of the observations list, available only in electronic form at the CDS.

\begin{tabular}{cccc}
\hline \hline Date & Right ascension & Declination & Telescope \\
\hline 2452498.4416123 & 254.7611902 & -12.8015470 & 1.60 \\
2452498.4423079 & 254.7611810 & -12.8015524 & 1.60 \\
2452874.4276516 & 256.9891248 & -13.6677386 & 0.60 \\
\hline
\end{tabular}

Note. Starting from left to right column we have: the time in Julian Date (UTC); the right ascension and declination coordinates, given in degree and fraction; and the telescope utilized in the observation are shown. These topocentric observed positions of 134340 Pluto are referred to a mean equator and equinox J2000 system.

Table 4. Observed minus calculated statistics for 134340 Pluto observations.

\begin{tabular}{|c|c|c|c|c|}
\hline Telescopes & $\begin{array}{c}\overline{(\mathrm{O}-\mathrm{C})_{\alpha}} \\
\sigma_{\alpha}\end{array}$ & $\begin{array}{c}\overline{(\mathrm{O}-\mathrm{C})_{\delta}} \\
\sigma_{\alpha}\end{array}$ & $N_{\text {obs }}$ & $\bar{N}_{\text {stars }}$ \\
\hline $1.60 \mathrm{~m}$ & $\begin{array}{l}0.006 \\
0.025\end{array}$ & $\begin{array}{l}0.007 \\
0.021\end{array}$ & 641 & 22 \\
\hline $0.60 \mathrm{~m}$ & $\begin{array}{l}0.018 \\
0.040\end{array}$ & $\begin{array}{l}0.015 \\
0 .^{\prime} 038\end{array}$ & 467 & 84 \\
\hline Final result & $\begin{array}{l}0.011 \\
0.033\end{array}$ & $\begin{array}{l}{ }^{\prime} .010 \\
0^{\prime} 030\end{array}$ & 1108 & 48 \\
\hline
\end{tabular}

ascension, and declination coordinates are given in degree and fraction, with seven significant digits. Table 3 gives a sample of the electronic list.

\section{Results and concluding remarks}

This work has presented 1108 observed positions of 134340 Pluto made with two telescopes and distributed over 49 nights between 1995 and 2004. These positions were compared to their theoretical positions, which are available electronically from the JPL Horizons on line solar system data and ephemeris computation service (Giorgini et al. 1996).

To evaluate the accuracy of observations, Table 4 shows the results of this paper. The ${\overline{(\mathrm{O}-\mathrm{C})_{\alpha}}}_{\text {and }}{\overline{(\mathrm{O}-\mathrm{C})_{\delta}}}_{\delta}$ are the residual means, observed minus calculated right ascension and declination coordinates, and $\sigma_{\alpha}$ and $\sigma_{\delta}$ are the standard deviation of residuals. The number of observations accomplished with each telescope and the mean number of star's CCD fields, used for the astrometric calibration, are shown in the last two columns. In Table 4 the results $\overline{(\mathrm{O}-\mathrm{C})_{\alpha}}, \overline{(\mathrm{O}-\mathrm{C})_{\delta}}$ and $\sigma_{\alpha}, \sigma_{\delta}$ are the mean and the standard deviations of the topocentric coordinates residuals (units in arcseconds). $N_{\text {obs }}$ is the number of observations and $\bar{N}_{\text {stars }}$ the mean number of stars' reference used in the astrometric calibration process.

The final results, presented in Table 4, enable us to obtain a very small dispersion in the position of 134340 Pluto. The residuals obtained with the $1.60 \mathrm{~m}$ telescope observations are twice better than those obtained with the $0.60 \mathrm{~m}$ telescope observations. However, both results show the good agreement with theoretical position. The results are within the expected ephemeris precision. These differences arise from the systematic errors in the classical method of astrometric calibration. These errors are caused by a non-uniform distribution of stars field. Still, these systematic errors also proceed from the reference catalog.

Figure 2 presents the residuals of 134340 Pluto as a function of time. We can see that the distribution of the residuals is uniform over these nine years. 

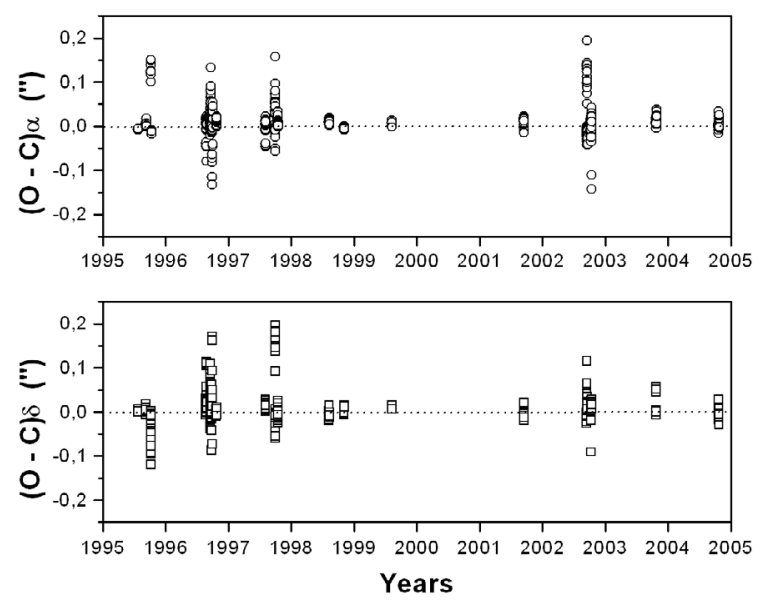

Fig. 2. Residuals $(\mathrm{O}-\mathrm{C})_{\alpha}$ and $(\mathrm{O}-\mathrm{C})_{\delta}$, in arcseconds, for the 134340 Pluto as a function of time.

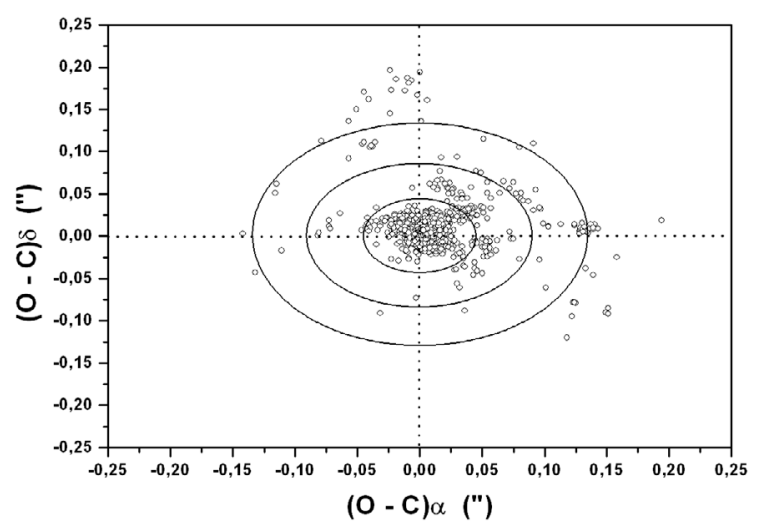

Fig. 3. $(\mathrm{O}-\mathrm{C})_{\alpha}$ versus $(\mathrm{O}-\mathrm{C})_{\delta}$, observed minus calculated positions in the right ascension and declination directions (in arcseconds). The $1 \sigma, 2 \sigma$, and $3 \sigma$ are represented by concentric ellipses outward from the origin.

In Fig. 3, the distribution of the residuals $(\mathrm{O}-\mathrm{C})_{\alpha}$ versus $(\mathrm{O}-\mathrm{C})_{\delta}$ are shown. The area where the residuals are contained is limited by concentric ellipses, whose semi-major axis and semi-minor axis are calculated by the standard deviations of mean residuals in right ascension and declination, respectively. For example, we can observe a cluster of 1017 residuals
Table 5. Distribution of residuals within $1 \sigma, 2 \sigma$, and $3 \sigma$.

\begin{tabular}{cc|c}
\hline \hline \multicolumn{2}{c|}{ Number of residuals per $\sigma$} & Normal \\
\hline $1 \sigma$ & $897(80,96 \%)$ & $46 \%$ \\
$2 \sigma$ & $1017(91,79 \%)$ & $91 \%$ \\
$3 \sigma$ & $1067(96,30 \%)$ & $99 \%$ \\
\hline
\end{tabular}

very close to the origin, corresponding to $2 \sigma \leq 0$. .'09 (91.79\%). Analyzing the other values in Table 5, we can consider that all residuals distribution satisfy the normality condition.

Table 5 shows the number of residuals within $1 \sigma, 2 \sigma$, and $3 \sigma$, relative to 1108 positions of 134340 Pluto. The relative percentage is given in brackets. The percentages show that the residuals distribution are within the expected normal distribution.

The 134340 Pluto orbit is chaotic. The computer simulations show that, after intervals longer than the Lyapunov time of 10-20 million years, it is impossible to determine exactly where 134340 Pluto will be (Sussman et al. 1988; Wisdom et al. 1991). I therefore consider that these observations are part of an important series because they can give a small but significant contribution to the accuracy of the ephemeris and can help to know the origin and formation of this mysterious Trans-Neptunian system better.

Acknowledgements. The author thanks all the staff of the Laboratório Nacional de Astrofísica/MCT for their assistance and help during these observations. Also, the author wants to thank the State of Rio de Janeiro Science Foundation FAPERJ for providing computational facilities for this project.

\section{References}

Gingerich, O. 2006, The Path to Defining Planets, Harvard-Smithsonian Center for Astrophysics and IAU EC Planet Definition Committee chair Giorgini, J. D., et al. 1996, BAAS, 28, 25.04

Lawson, C. L., \& Hanson, R. J. 1974, Solving Least Squares Problems (Englewood Cliffs: Prentice-Hall)

Monet, D., Bird, A., et al. 1998, The USNO-A2.0 Catalogue, Washington DC, US Naval Observatory

Null, G. W., Owen, W. M., Jr., \& Synott, S. P. 1993, AJ, 105, 2319

Reinsch, K., Burwitz, V., \& Festou, M. C. 1994, Icarus, 108, 209

Stern, S. A., Weaver, H. A., Steffl, A. J., et al. 2006, Nature, 439, 946

Stern, S. A., et al. 2007, Lunar and Planetary Science XXXVIII, 1722

Sussman, G. J., \& Wisdom, J. 1988, Science, 241, 433

Veiga, C. H., \& Vieira Martins, R. 1995, A\&AS, 111, 387

Weaver, H. A., Stern, S. A., Mutchler, M. J., et al. 2006, Nature, 439, 943

Wisdom, J., \& Holman, M. 1991, AJ, 102, 1528

Zacharias, N., Urban, S. E., Zacharias, M. I., et al. 2004, AJ, 127, 3043 